\title{
Coregistration of datasets from a micro-SPECT/CT and a preclinical 1.5 T MRI
}

\author{
J.-P. Dillenseger ${ }^{a}$, B. Guillaud ${ }^{b}$, C. Goetz ${ }^{a, c}$, A. Sayeh ${ }^{a}$, R. Schimpf ${ }^{b}$, A. Constantinesco ${ }^{a}$, P. Choquet $^{a, c, *}$ \\ a UF6237 Preclinical Imaging Laboratory, Hôpitaux Universitaires de Strasbourg, CHU Hautepierre, 1, Avenue Molière, 67098 Strasbourg, France \\ ${ }^{\mathrm{b}}$ RS2D, 24, Rue des Couturières, 67240 Bischwiller, France \\ ' Institut de Mécanique des Fluides et des Solides, CNRS, 2 rue Boussingault, 67000 Strasbourg, France
}

\section{A R T I C L E I N F O}

Keywords:

Small animal imaging

MRI

Micro-SPECT

Micro-CT

Multimodality

Coregistration

\begin{abstract}
A B S T R A C T
An universal tool was designed for small animal SPECT/CT/MR coregistration. It was tested on a preclinical MRI (OPTImouse, RS2D, Bischwiller, France) and a micro-SPECT/CT (eXplore speCZT Vision 120, GE, Waukesha, USA), closed to each other, thanks to the short extension of the MRI magnet fringe field. The tool consists of a curved catheter describing many rigid loops, and fixed on a plastic sheet. During acquisitions, it is placed around the animal, in an isolated imaging cell, and filled with a solution containing iodine, copper sulfate and radioisotope. Multimodality imaging is achieved sequentially by moving the cell from one system to the other, in about $20 \mathrm{~s}$. Acquisitions on phantom demonstrate the resolution accuracy of the coregistration process. Whole body trimodal SPECT/CT/MR acquisitions on live mice were coregistrated as well. A simple, cheap tool, easy to fill, could efficiently help for rigid coregistration of preclinical images, acquired on separate imaging apparatus.
\end{abstract}

(c) 2012 Elsevier B.V. All rights reserved.

\section{Introduction}

Combined multimodality instruments have already demonstrated their interest, both in clinical and preclinical fields. Due to the richness in soft tissues contrast, it was stated that coupling scintigraphic modalities with MRI will overcome the advantages of CT [1,2]. However, it is more challlenging and costly to combine any instrument with MRI due to magnetism, especially at high magnetic fields. There are 3 described possibilities for coupling scintigraphy and MRI [3]: together in line, PET or SPECT insert placed into a standard MRI system and a completely new built dual-system. As not all applications require the necessity of applying imaging protocols at the same time on both modalities, using different apparatus remains a solution for multimodality with MRI, especially for small animal imaging [3,4]. The feasibility of this last configuration must fulfill 3 conditions: imaging system should be present in a restricted area [5], place the animal in the same position between systems [6], a registration system to make fusion achievable $[7,8]$. Using a $1.5 \mathrm{~T}$ preclinical MRI system, a micro-SPECT/CT and the same imaging cell, moved between instruments, we propose a simple tool, to facilitate coregistration.

\footnotetext{
* Corresponding author at: UF6237 Preclinical Imaging Laboratory, Hôpitaux Universitaires de Strasbourg, CHU Hautepierre, 1, Avenue Molière, 67098 Strasbourg, France. Tel.: +33 3881167 68; fax: +33388128342.

E-mail address: pchoquet@unistra.fr (P. Choquet).
}

\section{Material and methods}

\subsection{MRI system and acquisition parameters}

MRI system (OPTImouse, RS2D, Bischwiller, France) uses a $1.5 \mathrm{~T}$ cryogen-free superconducting magnet. It takes up a floor area of $1.20 \mathrm{~m} \times 2 \mathrm{~m}$ while the $5 \mathrm{G}$ line is at $80 \mathrm{~cm}$ away from the center of the magnet in the worst case. A RF coil (Rapid Biomedical, Würzburg, Germany) of $40 \mathrm{~mm}$ in diameter is used, which remains inside the magnet. Phantom acquisition used a $8 \mathrm{~min} 12 \mathrm{~s}$ 3D $\mathrm{T} 1$ weighted spoiled gradient recalled echo sequence with isotropic voxels of $500 \times 500 \times 500 \mu \mathrm{m}^{3}$. The time was increased to $17 \mathrm{~min}$ for in vivo imaging.

\subsection{SPECT-CT system and acquisition parameters}

The micro-SPECT/CT (eXplore speCZT Vision 120, GE, Waukesha, USA) is installed about $2 \mathrm{~m}$ away from the MRI, thanks to the short extension of the magnet fringe field. Micro-CT data are obtained at $70 \mathrm{kV}, 32 \mathrm{~mA}$ and $16 \mathrm{~ms}$ in $1.5 \mathrm{~min}$ for isotropic voxels of $100 \times 100 \times 100 \mu \mathrm{m}^{3}$. For imaging whole body of the mouse, it takes $4.5 \mathrm{~min}$ to cover the field of view. Helical SPECT acquisition with a 7 pinhole collimators lasted about 20 min giving reconstructed isotropic voxels of $330 \times 330 \times 330 \mu \mathrm{m}^{3}$.

\subsection{Fiducial coregistration tool}

A catheter (external diameter: $1.1 \mathrm{~mm} /$ inner diameter: $0.64 \mathrm{~mm}$ ) was fixed on a flexible plastic sheet, placed inside the anesthesia bed 

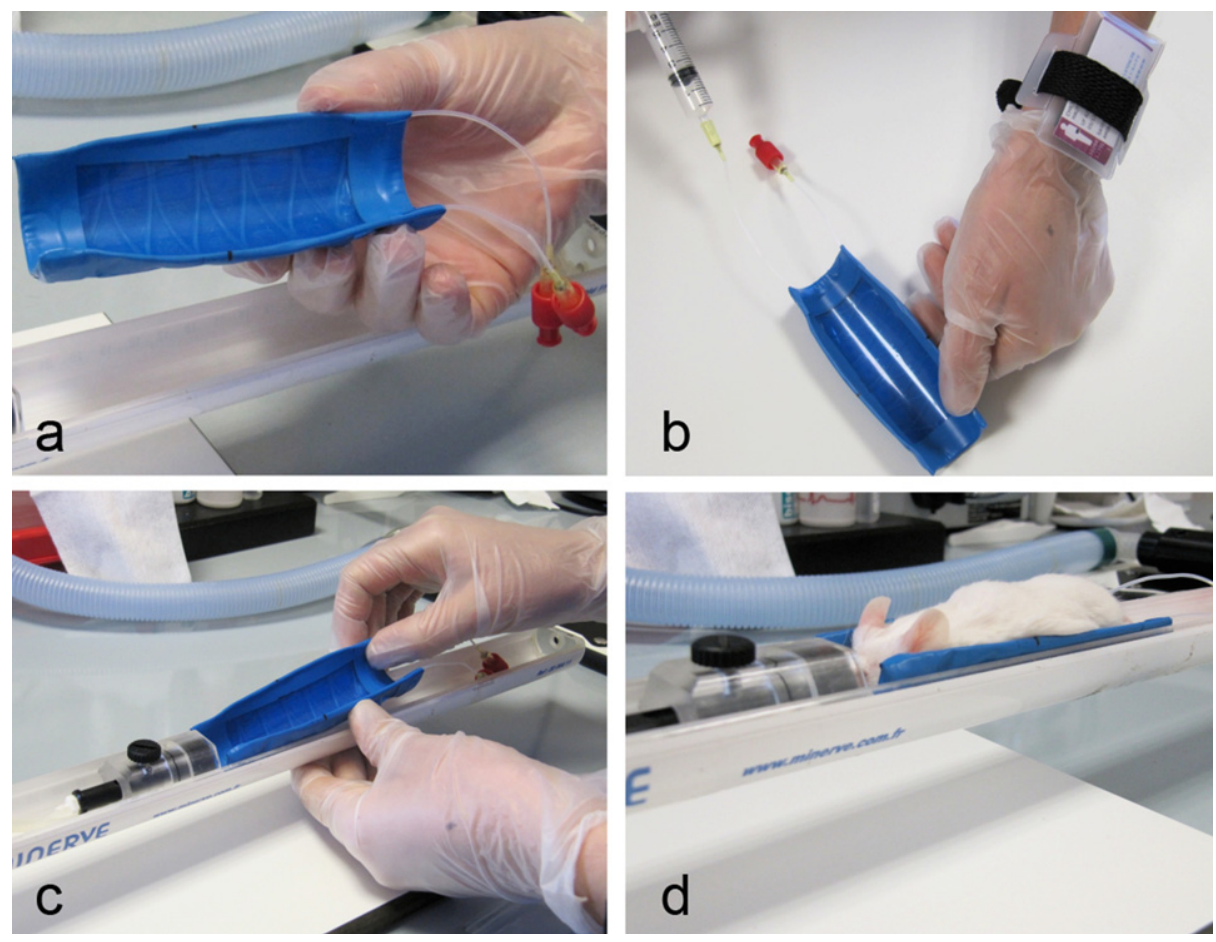

Fig. 1. The coregistration system tool (a). It takes less than $20 \mathrm{~s}$ to fill (b) and insert the tool in the imaging cell (c) and final configuration with an animal (d).

(Fig. 1): the half-pipe geometry of the system was adapted to the animal bed (for mice). The curved catheter described many rigid loops in order to cover each space directions to make 3D point based registration achievable. It was filled with a "multimodal solution" containing $50 \%$ iodine at $300 \mathrm{mgI} / \mathrm{mL}$ for CT $\left(\right.$ Omniscan $^{\mathbb{R}}$, GE Healthcare, Little Chalfont, UK), 50\% water with copper sulfate $(2 \mathrm{~g} / \mathrm{l})$ for MRI and radioisotope $(1 \mathrm{mCi} 99 \mathrm{mTc}$ ) for SPECT.

\subsection{Animal anesthesia and imaging cell}

Mice (C57B16) were kept anesthetized (air and 1\% isoflurane) and warmed in an imaging cell of inner diameter equal to $36 \mathrm{~mm}$ (Minerve, Esternay, France) during acquisitions. Multimodality imaging was achieved sequentially between the micro-SPECT/CT and the MRI by moving the cell from one system to the other, which takes approximately $20 \mathrm{~s}$ (Fig. 2).

\subsection{Test object}

In order to evaluate this registration system, we used a simple phantom which contains a PVC cylinder (diameter: $21 \mathrm{~mm} /$ length: $10 \mathrm{~mm}$ ) through which 2 crossing tubes (diameter: $1.25 \mathrm{~mm}$ ) were bored with an intersection angle of $54^{\circ}$. The directions of these 2 tubes are not parallel to any axis $x, y$ of the magnet (Fig. 3). For multimodal detection, the 2 tubes were filled with iodine (Omniscan $\left.{ }^{\circledR} 300 \mathrm{mgI} / \mathrm{mL}\right)$, copper sulfate $(2 \mathrm{~g} / \mathrm{L})$ and radioisotope (2 $\mathrm{mCi} 99 \mathrm{mTc}$ ).

\subsection{Image registration}

The point based registration comprises of several steps. First, choose and place registration points on the fiducial system ( 8 in our case). We used Amide (http://amide.sourceforge.net/) for computing the parameters of the rigid transformation which are achieved by a translation vector and a rotation matrix. Finally, integration of the transformed SPECT with the MRI or/and CT was conducted for image fusion.

For analyzing the accuracy of our point based registration system we used the fiducial registration error (FRE) and the target registration error (TRE). FRE is the root mean square distance between corresponding fiducial points after registration and TRE is the distance measure after registration between a reference corresponding point other than the fiducial points [10]. We considered this corresponding point as the intersection of the cross of our phantom. For statistical robustness, analyses were done on 6 different acquisitions.

\section{Results}

Table 1 presents results of the fiducial registration and target registration errors measured on 6 acquisitions between SPECT/ MRI, SPECT/CT and CT/MRI registrations. A total of 48 (8 landmarks $\times 6$ acquisitions) independent calculations were carried out for each coregistration possibility: SPECT/MRI, SPECT/CT and CT/ MRI. Mean values as well as standard deviations and also trimodal values are reported in Table 1.

In-vivo applications: whole-body image fusions of mice were obtained by using the registration tool, one result is shown in Fig.4.

\section{Discussion}

Our challenge was to design a coregistration tool that could be put inside the imaging cell between the animal and the bed $[8,9]$ and that could be detected in 3 modalities: CT, SPECT and MRI. It is fast enough and does not extend our protocols duration. Moreover, this low time manipulation is an advantage in terms of operator radioprotection. 

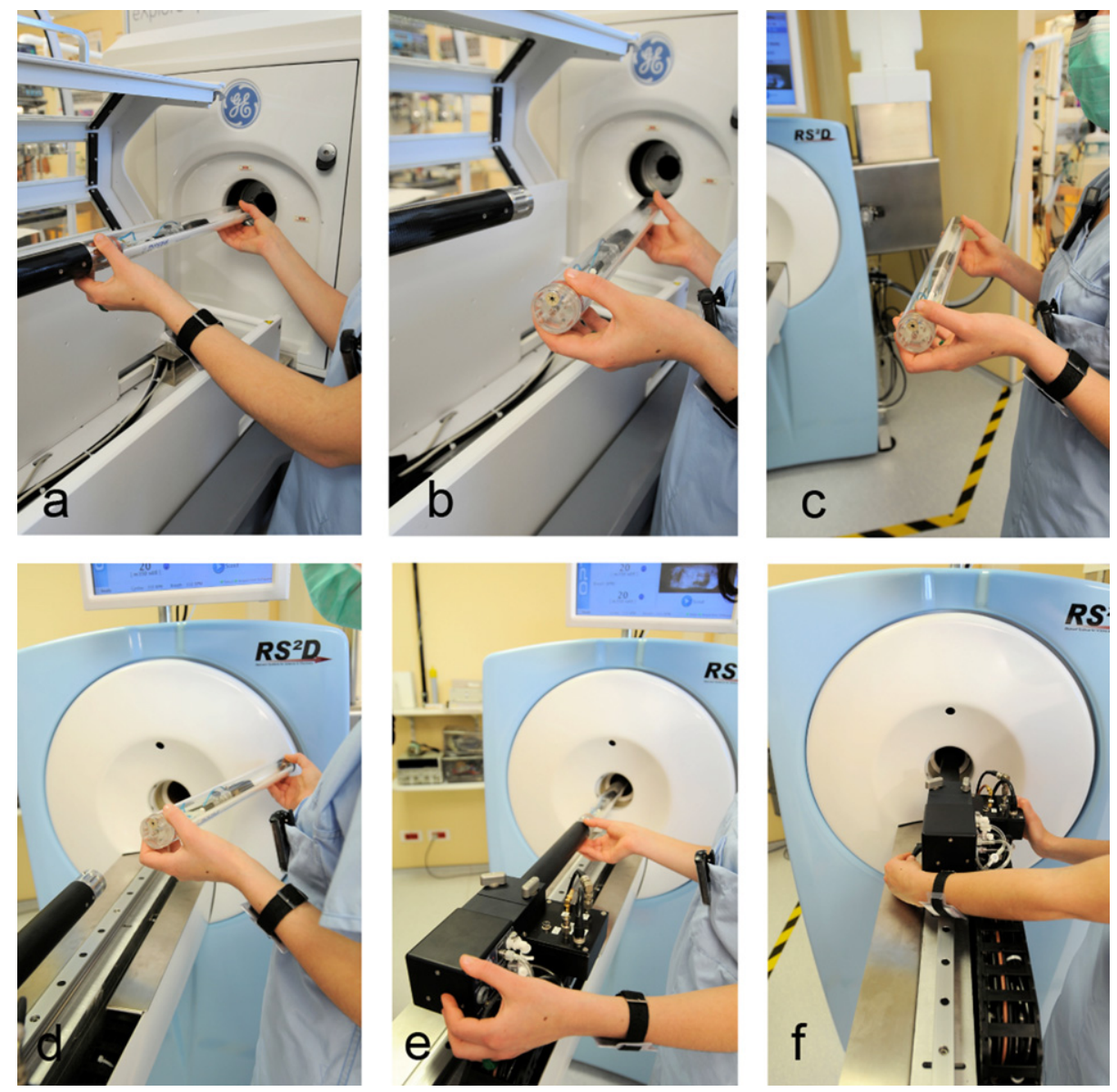

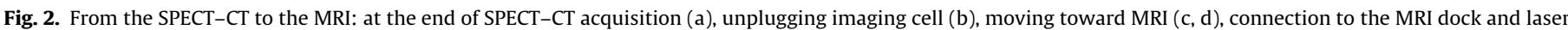
positioning (e), positioning inside the magnet (f) and beginning of MRI acquisition.
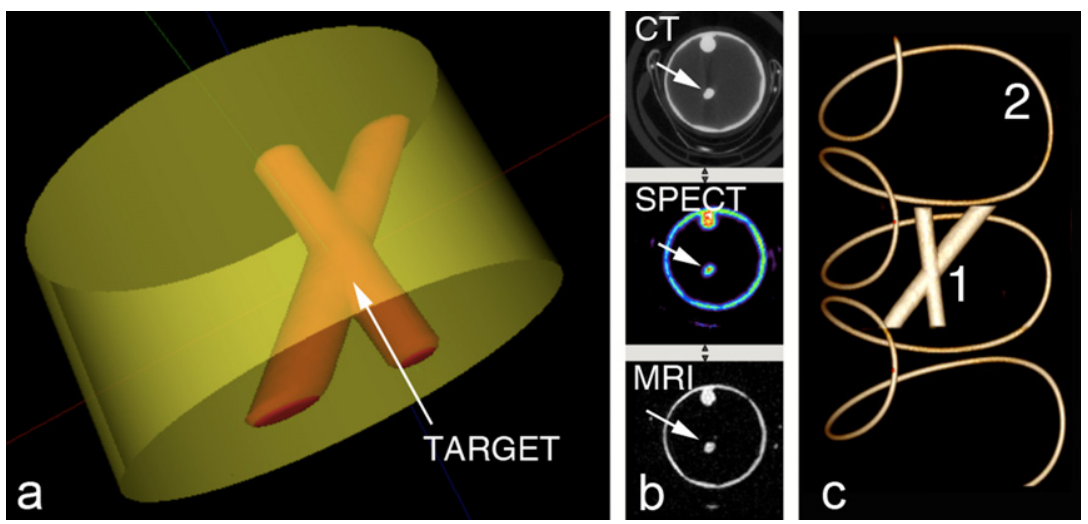

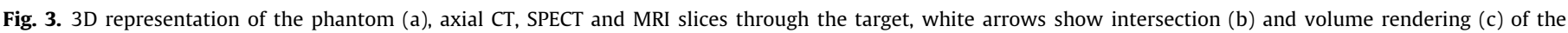
phantom (1) inside the registration tool (2), based on micro-CT data.

Table 1

Fiducial registration errors and target registration errors (in $\mathrm{mm}$ ).

\begin{tabular}{lllll}
\hline & SPECT-MRI & SPECT-CT & CT-MRI & \\
\hline FRE & & & & \\
Mean & 0.66 & 0.47 & 0.51 & 0.55 \\
Std. & 0.09 & 0.08 & 0.08 & 0.08 \\
TRE & & & & \\
Mean & 0.43 & 0.34 & 0.34 & 0.37 \\
Std. & 0.17 & 0.17 & 0.15 & 0.16 \\
\hline
\end{tabular}

\section{Conclusion}

We have demonstrated that a simple, cheap tool, easy to fill, could efficiently help for rigid coregistration of preclinical images, acquired on separate imaging apparatus. The simplicity of the concept allows for an easy and quick setup, without wasting time and could be applied on other instruments than the ones used in this work. Results on phantoms demonstrate that the coregistration is adequate, showing in small animals that merging scintigraphic or/and CT data with MRI could be done even without a combined acquisition system. 


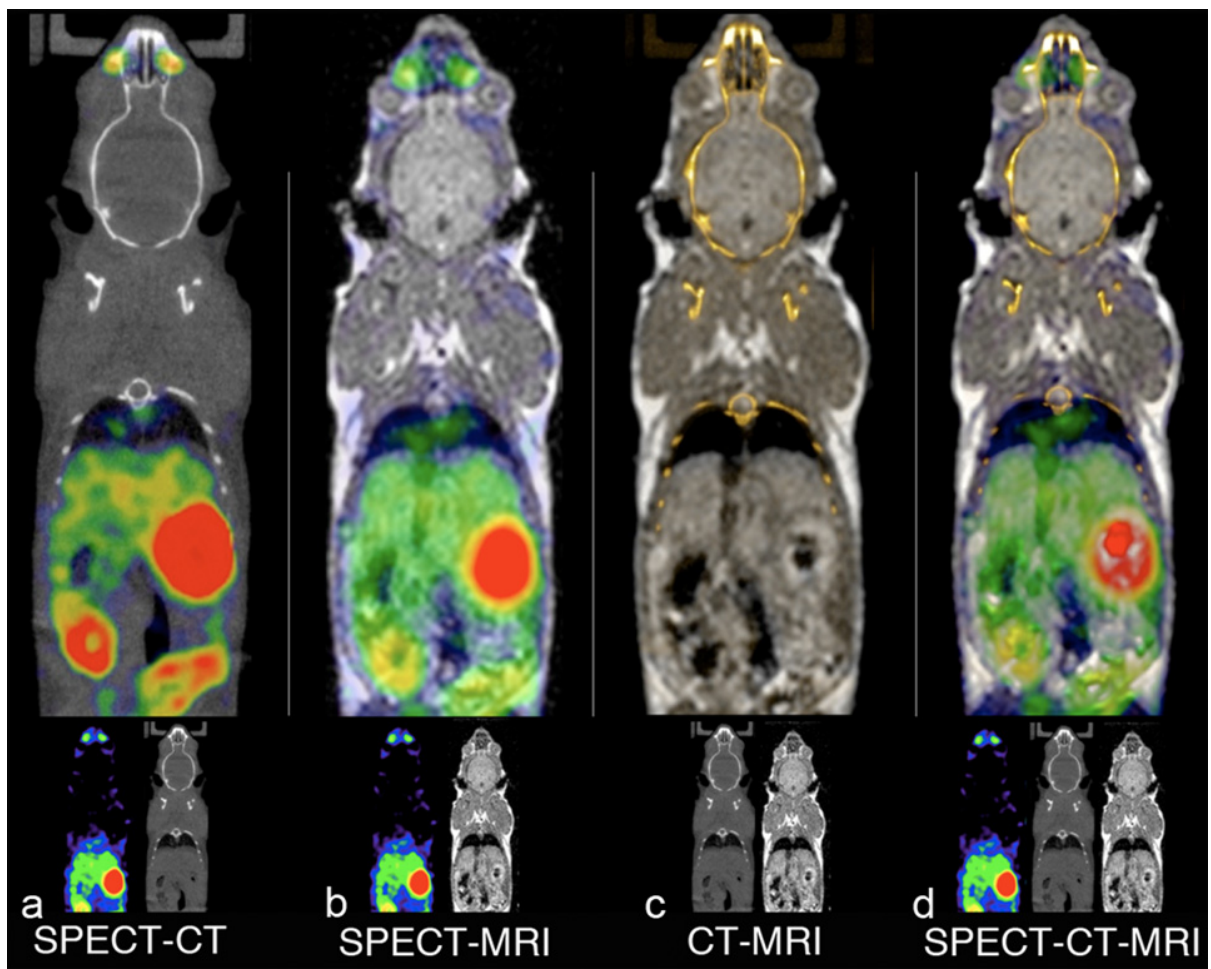

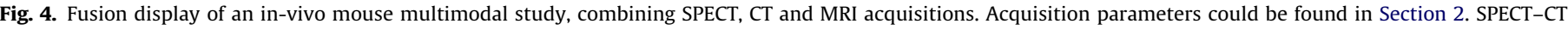
(a) uses the registration tool provided with the system while SPECT-MRI (b), CT-MRI (c) and SPECT-CT-MRI (d) are coregistrated using Amide.

\section{References}

[1] B.J. Pichler, H.F. Wehrl, A. Kolb, M.S. Judenhofer, Seminars in Nuclear Medicine 38 (2008) 199.

[2] A.W. Sauter, H.F. Wehrl, A. Kolb, M.S. Judenhofer, B.J. Pichler, Trends in Molecular Medicine 16 (2010) 508.

[3] M. Zhang, M. Huang, C. Le, P.B. Zanzonico, F. Claus, K.S. Kolbert, K. Martin C.C. Ling, J.A. Koutcher, J.L. Humm, Physics in Medicine and Biology 53 (2008) 5867.

[4] C.M. McCann, P. Waterman, J.-L. Figueiredo, E. Aikawa, R. Weissleder, JW. Chen, Neuroimage 45 (2009) 360.

[5] C. Goetz, E. Breton, P. Choquet, V. Israel-Jost, A. Constantinesco, Journal of Nuclear Medicine 49 (2008) 88.
[6] C. Ji, F. van der Have, H. Gratama van Andel, R. Ramakers, F. Beekman International Journal of Biomedical Imaging 2010 (2010) 654506.

[7] M. Nahrendorf, E. Keliher, B. Marinelli, P. Waterman, P.F. Feruglio, L. Fexon, M. Pivovarov, F.K. Swirski, M.J. Pittet, C. Vinegoni, R. Weissleder, Proceedings of the National Academy of Sciences of the United States of America 107 (2010) 7910.

[8] T.S. Ng, D. Procissi, Y. Wu, R.E. Jacobs, Medical Physics 37 (2010) 1995.

[9] C.C. Yang, T.H. Wu, M.H. Lin, Y.H. Huang, W.Y. Guo, C.L. Chen, T.C. Wang, W.H. Yin, J.S. Lee, Nuclear Instruments and Methods in Physics Research Section A 569 (2006) 240.

[10] R.F. Labadie, R.J. Shah, S.S. Harris, E. Cetinkaya, D.S. Haynes, M.R. Fenlon, A.S. Juscyzk, R.L. Galloway, J.M. Fitzpatrick, Computer Aided Surgery 9 (2004) 145. 